\title{
Gaussian beam evolution in logarithmically saturable nonlinear media
}

\author{
P. Berczyński, ${ }^{* 1}$ J. Jasiński ${ }^{2}$, Yu. Kravtsov ${ }^{3}$ \\ ${ }^{1}$ Institute of Physics, West Pomeranian University of Technology, Szczecin 70-310, Poland, \\ ${ }^{2}$ Faculty of Physics, Warsaw University of Technology, Koszykowa 75, 00-662 Warszawa \\ ${ }^{3}$ Institute of Physics, Maritime University of Szczecin, Szczecin 70-500, Poland
}

Received May 11, 2013; accepted June 16, 2013; published June 30, 2013

\begin{abstract}
The method of paraxial complex geometrical optics (PCGO) is presented, which describes Gaussian beam (GB) diffraction and self-focusing in smoothly inhomogeneous and nonlinear media of cylindrical symmetry. PCGO reduces the problem of Gaussian beam diffraction in nonlinear and inhomogeneous media to the system of the first order ordinary differential equations for the complex curvature of the wave front and for GB amplitude, which can be readily solved both analytically and numerically. The power of the PCGO method is exemplified by GB evolution in a logarithmically saturable medium with a defocusing refractive profile. The solutions obtained by the PCGO method are compared with numerical results of a Nonlinear Schrodinger Equation by the beam propagation method (BPM).
\end{abstract}

Complex geometrical optics (CGO) has two equivalent forms: the ray-based form, which deals with complex rays [1-6], that is with trajectories in a complex space, and the eikonal-based form, which uses complex eikonal instead of complex rays [6,7]. A surprising feature of CGO is its ability to describe Gaussian beam (GB) diffraction in both ray-based and eikonal-based approaches. Recently, eikonal-based CGO method has been applied to describe GB evolution in inhomogeneous media [8,9], nonlinear inhomogeneous fibres [10] and graded-index fibres [11]. It is shown in [11] that eikonal-based CGO approach demonstrates high ability to describe GB evolution in graded-index optical fibres reducing hundred times the time of numerical calculations at comparable accuracy with a Crank-Nicolson scheme in the Beam Propagation Method (BPM).

This paper describes the advantages of the eikonalbased form of PCGO for the description of Gaussian beam diffraction and self-focusing in a logarithmically saturable medium with defocusing refraction. This paper generalizes the results of previous papers [12-14], where the authors considered mainly light beam propagation in nonlinear saturable media without any contribution of the linear refraction. Although media with logarithmic nonlinearity have not been encountered in nonlinear optics, it has been argued that "accessible" nonlinearities of various forms (including a logarithmic one) can "provide a valuable insight and still maintain the characteristic features of the underlying physical process" [14].

\footnotetext{
*E-mail: pawel.berczynski@zut.edu.pl
}

For an axially symmetric wave beam in an axially symmetric nonlinear medium, the PCGO method suggests a solution of the form

$u(r, z)=A \exp \left(i k_{0} \psi\right)=A(z) \exp \left[i k_{0}\left(z+B(z) r^{2} / 2\right)\right] .(1)$

The real and imaginary parts of the complex curvature $B=B_{R}+i B_{I}$ determine the real curvature $\kappa$ of the wave front and the beam width $w$ correspondingly:

$$
B_{R}=\kappa, \quad B_{I}=\frac{1}{k_{0} w^{2}},
$$

where $k_{0}=2 \pi / \lambda_{0}$ and $\lambda_{0}$ is the wavelength of the beam in vacuum. The PCGO method deals with the Riccati equation for complex curvature $\mathrm{B}$ :

$$
\frac{d B}{d z}+B^{2}=\alpha
$$

equation for GB complex amplitude

$$
\frac{d A}{d z}+B A=0 .
$$

and the second order equation for GB width evolution,

$$
\frac{d^{2} w}{d z^{2}}-\alpha w=\frac{1}{k_{0}^{2} w^{3}}
$$

Parameter $\alpha$ for axially symmetric medium equals:

$$
\alpha=\left.\frac{1}{2} \frac{\partial^{2} \varepsilon}{\partial r^{2}}\right|_{r=0} .
$$

Above PCGO equations are derived in paper [10].

The PCGO method is applied in this paper for GB propagation in inhomogeneous defocusing and a cylindrically symmetric nonlinear medium with a permittivity profile of the form 


$$
\varepsilon=\varepsilon_{0}+g r^{2}+\varepsilon_{N L} \ln \left(I / I_{s a t}\right),
$$

where $I=u u^{*}$ is the beam intensity, $r$ is the distance from the fibre axis $\mathrm{z}, g$ is the graded-index coefficient and $I_{s a t}$ is the characteristic saturation intensity. The logarithmic nonlinear dependence presented in Eq. (7) is approximation of the saturable model $\ln \left(1+I / I_{\text {sat }}\right)$ for $I>>I_{s a t}$. For the refractive index (7), the Riccati equation (3) takes the form

$$
\sqrt{\varepsilon_{0}} \frac{d B}{d z}+B^{2}=-\frac{\varepsilon_{N L}}{w^{2}}+g
$$

and equation for GB width evolution (5) is in the form:

$$
\varepsilon_{0} \frac{d^{2} w}{d z^{2}}+\frac{\varepsilon_{N L}}{w}-g w=\frac{1}{k_{0}^{2} w^{3}} .
$$

Introducing next dimensionless GB width $f=w / w_{0}$, where $w_{0}=w(0)$ denotes the initial beam width, one obtains the equation:

$$
\varepsilon_{0} \frac{d^{2} f}{d z^{2}}+\frac{\varepsilon_{N L}}{w_{0}^{2} f}-g f=\frac{1}{k_{0}^{2} w_{0}^{4} f^{3}} .
$$

Introducing normalized variables $R, Z$ and normalized material parameters $G$ and $N$ :

$$
\begin{gathered}
R=\frac{r}{w_{0}}, \quad Z=\frac{z}{k w_{0}^{2}}, \\
G=k_{0}^{2} w_{0}^{4} \cdot g, \quad N=k_{0}^{2} w_{0}^{2} \cdot \varepsilon_{N L},
\end{gathered}
$$

Eq. (10) takes the form

$$
\frac{d^{2} f}{d Z^{2}}+\frac{N}{f}-G f=\frac{1}{f^{3}} .
$$

Demanding next for the beam to propagate as a stationary mode in a logarithmically nonlinear medium with defocusing refraction we put $f=1$ in Eq. (12) obtaining the condition:

$$
G=N-1 \text {. }
$$

Let us compare PCGO results with numerical and analytical solutions of a Nonlinear Schrodinger Equation (NLS). A nonlinear parabolic equation for the case of axially symmetric beam in axially symmetric medium has the form:

$$
\frac{\partial u}{\partial z}=\frac{i}{2 k} \frac{1}{r} \frac{\partial}{\partial r}\left(r \frac{\partial u}{\partial r}\right)+i k\left(1+\frac{g r^{2}}{2 \varepsilon_{0}}+\frac{\varepsilon_{N L} \cdot \mathrm{Y}\left(|u|^{2}\right)}{2 \varepsilon_{0}}\right) u,
$$

where $\mathrm{Y}\left(|u|^{2}\right)$ is the nonlinear function. In a logarithmically saturable medium this function for $|u|^{2}>>I_{\text {sat }}$ is

$$
\mathrm{Y}\left(|u|^{2}\right)=\ln \left(|u|^{2} / I_{\text {sat }}\right) .
$$

Taking into account Eq. (15), the nonlinear parabolic equation takes the form

$$
\frac{\partial u}{\partial Z}=\frac{i}{2} \frac{1}{R} \frac{\partial}{\partial R}\left(R \frac{\partial u}{\partial R}\right)+i\left(k^{2} w_{0}^{2}+\frac{G R^{2}}{2}+\frac{N \mathrm{Y}\left(|u|^{2}\right)}{2}\right) u .
$$

For Y given by Eq. (15,) we search for the solutions of Eq. (16) in the form

$$
u=\sqrt{I_{0}} \cdot \Psi \cdot \exp \left(i\left(k_{0}^{2} w_{0}^{2}-\frac{N}{2} \ln \frac{I_{s a t}}{I_{0}}\right) Z\right)
$$

wh gives Nonlinear Schrodinger Equation (NLS) for the complex envelope $\Psi=\Psi(R, Z)$ in the form:

$$
\frac{\partial \Psi}{\partial Z}=\frac{i}{2} \frac{1}{R} \frac{\partial}{\partial R}\left(R \frac{\partial \Psi}{\partial R}\right)+\frac{i}{2}\left(G R^{2}+N \ln \left(|\Psi|^{2}\right)\right) \Psi .
$$

One can prove that NLS in Eq. (18) possesses an analytical solution in the form of a Gaussian beam:

$$
\Psi=\frac{1}{f(Z)} \exp \left(-\frac{R^{2}}{2 f^{2}(Z)}+\frac{i R^{2} f^{\prime}(Z)}{2 f(Z)}+i \Delta \Phi(Z)\right),
$$

where:

$$
\Delta \Phi(Z)=-i \int_{0}^{Z}\left(N \ln f(\xi)+\frac{1}{f^{2}(\xi)}\right) d \xi .
$$

and provided that parameter f satisfies Eq. (12).

One can notice that the equations for GB width evolution obtained by the PCGO method and the NLS method are identical and are presented in Eq. (12). This way the PCGO method provides exact solutions of NLS for logarithmically saturable nonlinear media modeled by Eq. (7). This is illustrated in Fig. 1 and Fig. 2 where PCGO solutions are compared with numerical results of NLS obtained by BPM for the nonlinear parameter $N=0.4 \pi^{2}=$ 3.94784 . 


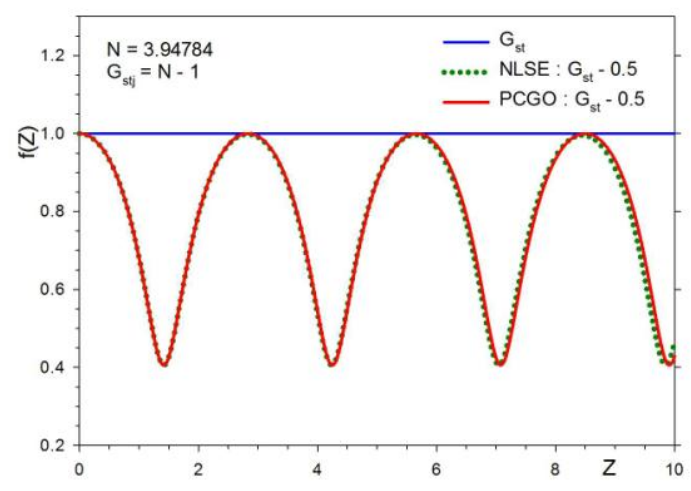

Fig. 1. Comparison of PCGO oscillatory solution with numerical results of BPM for $w_{0}=10 \lambda$ and $\varepsilon_{N L}=10^{-3}$.

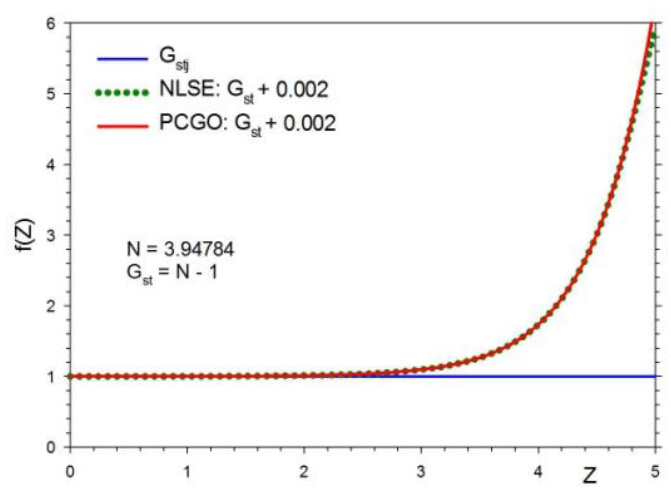

Fig. 2. Comparison of PCGO diffractional solution with numerical results of BPM for $w_{0}=10 \lambda$ and $\varepsilon_{N L}=10^{-3}$.

In Fig. 3, at the phase plane, we present the possible types of solutions of Eq. (12) for GB width evolution $f$. The phase curves $f^{\prime}(f)$ are drawn for the previously assumed nonlinear parameter $N$. The stationary solution of Eq. (12) obtained for $G$ satisfying the condition in Eq. (13) is given by the point lying at the center of figure $f=1, f^{\prime}=0$. One can notice that this solution is unstable because the small fluctuation $\delta f<0$ causes appearance of oscillations of GB width $f$ between $f_{\max }=1$ and certain $f_{\min }(N)$ (for $N=0.4 \pi^{2}$ we have $\left.f_{\min }=0.44782\right)$. On the other hand, the small fluctuation $\delta f>0$ leads to diffraction, where the GB width $f$ increases to infinity. For the graded-index coefficient $G<G_{\text {st }}=N-1$ we obtain stable oscillations, for which diffraction widening appears when initial width $f(0)$ is sufficiently greater. Similarly, $G>G_{\text {st }}$ causes diffraction of GB. However, the narrower beams could oscillate in such a medium (closed red curve in the left side of Fig. (3)).

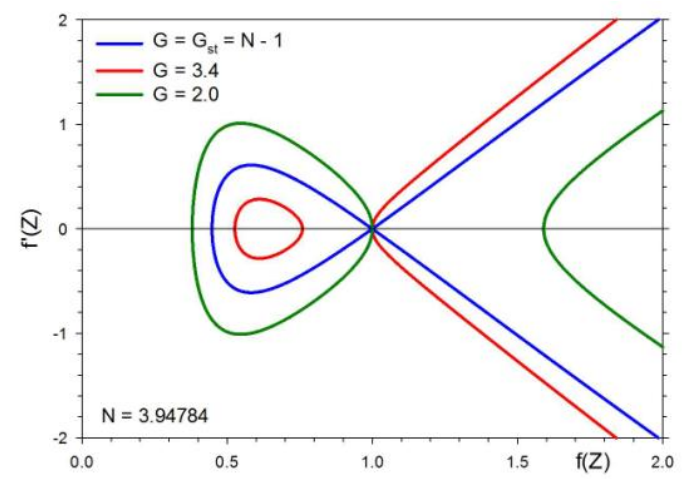

Fig. 3. PCGO and NLS solutions in the form of GB width $f$ evolution illustrated on the phase plane and obtained for a nonlinear saturable medium with permittivity in Eq. (7) .

\section{References}

[1] Yu.A. Kravtsov, Radiophys. Quantum Electronics 10, 719 (1967)

[2] J.B. Keller, W. Streifer, J. Opt. Soc. Am. 61, 40 (1971).

[3] G. A. Deschamps, Electron. Lett. 7(23), 684 (1971).

[4] Yu.A. Kravtsov, G.W. Forbes, A.A. Asatryan, Theory and applications of complex rays, in Progress in Optics, edited by E. Wolf, 39, 3 (Elsevier, Amsterdam, 1999).

[5] S.J. Chapman, J.M. Lawry, J.R. Ockendon, R.H. Tew, SIAM Review 41, 417 (1999).

[6] Yu.A. Kravtsov, P. Berczynski, Stud. Geophys. Geod. 51(1), 1 (2007).

[7] Yu.A. Kravtsov N.Y. Zhu. Theory of diffraction: Heuristic Approaches (Alpha Science International, ISBN 1842653725, 2009).

[8] P. Berczynski, Yu.A. Kravtsov, Phys. Lett. A 331(3-4), 265 (2004).

[9] P. Berczynski, K.Yu. Bliokh, Yu.A. Kravtsov, A. Stateczny, J. Opt. Soc. Am. A, 23(6), 1442 (2006).

[10] P. Berczyński, Yu. Kravtsov, Phot. Lett. Poland 4(1), 26 (2012).

[11] P. Berczynski, Yu. A. Kravtsov, G. Zeglinski, Optica Applicata 40(3), 705 (2010).

[12] A.W. Snyder, J.D. Mitchell, Optics Letters 22(1), 16 (1997).

[13] Y.L. Tang, J.G Chen, D.Y Li, J. Kang, Y. Li, J. Modern Optics 46(8), 1177 (1999).

[14] T. Hansson, D. Anderson, M. Lisak, Opt. Comm. 283(2), 318 (2010). 